\title{
VI.
}

Aus der k. k. chirurgisehen Klinik des Hofrats Prof.

Ludw. R. v. Rydy gier.

\section{Über die Endresultate der Radicaloperationen der Unterleibshrüche.}

\author{
Yon \\ I)r. Felix Hahn, \\ Regimentsarzt des k. k. Landwohr-Infanterie-Regiments Stanislaus Nr. $\mathbf{0}$, \\ abkommandiert zar obigen Klinik.
}

In den nachstehenden Zeilen soll über die Endresultate der an der Rydygiersehen Klinik, in der Zeit vom Wintersemester 1886 bis zum Sommersemester 1897 in Kraka und vom Sommersemester 1897 bis zum Wintersemester 1901 in I Lemberg, vorgenommenen Mernien-Radicaloperationen berichtet werden.

Es wird vielleicht auffallen, daß an der erwähnten Klinik eine für den Zeitraum von 15 Jahren verhältnismäßig geringe Zahl von Bruchkranken zur Aufnahme gelangte, doch erklärt sich dies aus dem Lmstande, daß wegen der geringen Belagsfähigkeit dieser $\mathbf{A n}$ stalt, sowie in dem Bestreben, den Studierenden die ausgiebigste Abwechslung zu bieten, nieht alle sich meldenden Kranken aufgenommen worden konnten, sondern die meisten mit Fremien behafteten Personen an andere Heilanstalten gewiesen werden mußten.

Während des oberwälnten Zeitraumes wurden 268 Bruchkranke (191 Männer und 77 Frauen) aufgenommen, u. z.: 1886-1897: 146, 1897-1901: 122 Personen.

Hiervon waren 242 mit je einer, 24 mit je $z w e i$ and 2 mit je drei IIernien behaftet.

Die doppelseitigen Brüche waren: in 20 Fällen je eine H. inguinalis bilateralis, in 2 Fällen je eine $H$. cruralis bilat., in 1 Falle eine H. inguinalis ext. und cine H. cruralis, schlieblich in 1 Falle eine $H$. cruralis und eine $\Pi$. ventralis. 
Über die Endresultate der Radicaloperationen dor Unterleibsbrüche. 123

Die mit je drei Hernien behafteten 2 Personen besaßen je eine H. inguinalis ext. bilat. und ein $\mathrm{H}$. epigastrica.

Dem Alter, in welchem die Bruchkranken operiert wurden, entsprechen folgende Daten:

$$
\begin{aligned}
& \text { unter } 5 \text { Jahren } 5 \text { Personen. }
\end{aligned}
$$

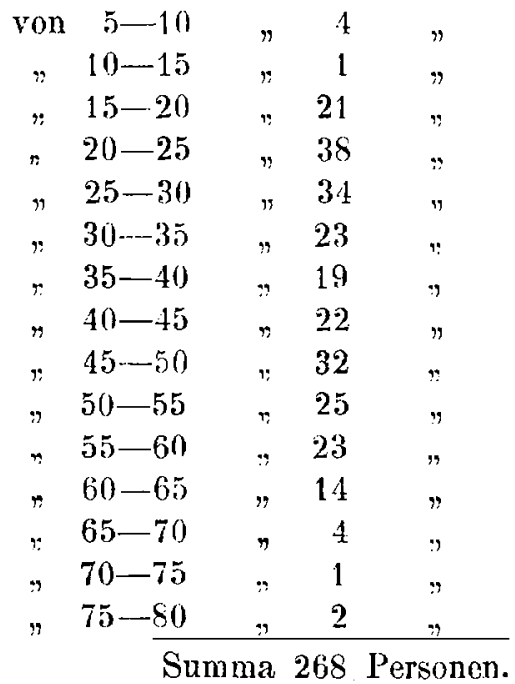

Die relativ große Zahl der Leibschäden im vorgerückten Alter (45.-60. Lebensjahr) findet in der Häufigkeit der in diesem. Alter vorkommenden Einklemmungen ihre Erklärung.' Solche Leute suchen nämlich hauptsächlich ihres gefahrdrohenden Zustandes wegen die Heilanstalt auf.

Von In carcerationen kamen vor:

\begin{tabular}{|c|c|c|}
\hline $\begin{array}{r}\text { unter } 10 \\
\text { von } 10-20\end{array}$ & $\begin{array}{cl}\text { ahren } & 4 \\
\eta & 6\end{array}$ & Fälle \\
\hline $20-30$ & 19 & " \\
\hline$" 30-40$ & 14 & , \\
\hline $40-50$ & 25 & \\
\hline$\Rightarrow \quad 50-60$ & 32 & . \\
\hline $60-70$ & 12 & . \\
\hline $70-80$ & 2 & " \\
\hline
\end{tabular}

Bezüglich der Religion wäre zu bemerken, daß rund 1; sämtlicher Patienten dem mosaischen Glauben angehörte.

Dem Berufe nach rekrutierten sich die Bruchkranken hauptsächlich aus den mittellosen, schwer arbeitenden Volksschichten. 

und zwar

Alles in allem wurden 290 Hernienoperationen ausgefübrt,

A. Herniae epigastrii: II. epigastricae lib. . . . . 4

B. Herniae mesogastrii:

C. Merniae hypogastrii:

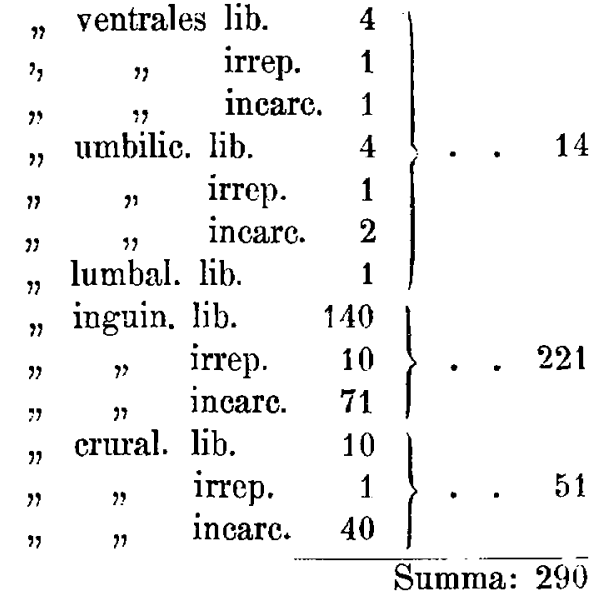

Dem Geschlechte nach kamen vor:

Herniae epigastrii bei 4 Männern, 0 Frauen,

$\begin{array}{llrlrl}" & \text { mesogastrii } & 2 & & 12 & \\ " & \text { inguinales } & 180 & & 23 & " \\ " \text { crurales } & 6 & & 43 & \end{array}$

Von den an der Klinik ausgeführten 237 Radical operationen sind bloß die Endresultate von 182 Fällen bekannt, und zwar sind 165 Dauererfolge, 16 Recidive, d. i. 8,8 Proz. und 1 Todesfall, d. i. 0,5 Proz. zu verzeichnen.

Als Erläuterung zu unseren weiteren Ausführungen schicken wir der Besprechung der einzelnen Hermienarten nachstehende, die einschlägigen Krankengeschichten in schlagwörtlicher Kürze enthaltende Tabellen voraus.

$$
\text { A. Brüche des Epigastriums. }
$$

Die ältesten Berichte über diese Bruchart stammen nach May dl $(5)^{1}$ ) von Richter [1785], während die Benennung derselben Vidal de Cassis [1848] zugeschrieben wird.

Die epigastrischen Hernien gehören zu den ziemlich seltenen Brucharten, und zwar entfallen nach Berger (6) von der Gesamtzahl verschiedener Brucharten ca. 1,4 Proz. auf epigastrische Hernien. Dieses Verhältnis trifft auch hier zu, da rom gesamten klinischen

1) Die in runden Klammern befindlichen Ziffern bezichen sich auf das am Schlusse angefügte Literaturverzeichnis. 
Über dic Endresultate der Radicalopcrationen der Lnterleibsbritiche. 125

Material 4 derlei Hernien auf 290 diverse entfallen; es unterliegt jedoch gar keinem Zweifel, daß diese Krankheitsform viel häufiger zur Beobachtung gelangen dïrfte, wenn man sich durch die ungenauen Angaben der Patienten und deren Klagen über allerband Hagenbeschwerden nicht beirren licße, sondern in jedem unklaren Falle die Banchwandungen grïndlich palpieren würde. Als Beweis dieser Behauptung können wir die Tatsache anführen, daß wir, angeregt durch Fall Nr. 4, der vicle "pralstische" Ärzte consultiert batte, bis sein Leiden erkannt wurde, bald darauf in rascher Aufeinanderfolge mehrere epigastrische Hernien zu diagnosticieren Gelegenheit batten, deren Besitzer indessen jeden operativen Eingriff verweigerten.

Die Diagnose der epigastrischen Hernien muß eben unbedingt gelingen, wenn man die schmerzhafte Bauchwandpartie, statt zu beklopfen, aufmerksam abtastet, während der Kranke aus der liegenden in die sitzende Position übergeht oder bei rubiger Rückenlage bustet.

Sämtliche 4 an unserer Klinik vorgekommene Fälle betrafen männliche Individuen, von denen zwei überdies noch mit je einer H. inguinalis externa bilateralis bebaftet waren. In einem der letzteren Fälle wurden alle 3 Hernien in einer Tarkose, in dem anderen dagegen wurde - wegen des bohen Niters - nur die ihrem Besitzer unerträgliche Beschwerden bereitende epigastrisehe Hernie operiert.

Das Operationsverfahren war in drei Fällen möglicbst einfach: Hautsehnitt, Bloblegung des Bruchsackes, Exstirpation desselben nach vorheriger Reposition des Inhaltes, Excision der. Brachpfortenränder, schließlich Naht der Bauchdecken, wie bei einer gewöhnlichen Laparotomie.

Fall Nr. 4 wurde (vom Verfasser) nach der Methode von Gersun y operiert: Hantschnitt, Bloßlegung des Bruchsackes, Reposition desselben (wegen seiner Kleinhcit), Auffrischung und Vernähung der Bruchpfortenränder, Spaltung beider Rectusscheiden mit leichter Convexität nach aufen in einer die Bruchpforte nach oben und unten um etwa $2 \mathrm{~cm}$ überragenden Länge und Vereinigung der inneren, dann der äußeren Schmittränder der Rectusseheide mittels Knopfnaht. - Nalıtmaterial: Catgut.

Die Heilung erfolgte in 3 Fällen per primam, in 1 Falle (Nr. 3), ist die Eiterung der Hautnähte darauf zurückzuführen, daß der Kranke, trotz strengsten Verbotes, dic Wunde unter dem Verbande mehrmals betastete.

Die Endresultate der Operation waren ziemlich günstige: 2 Daucrerfolge, 1 Recidiv (1 Fall ohne Nachricht). 


\section{B. Brüche des Mesogastriums.}

Die Briiche des Mesogastriums weisen 3 Arten auf: YabelLenden- und B auchbr ü che (Ventralhernien) im engeren Sinne.

Zu den letzteren zählt man alle jene IIernien, die sich an einer nicht näher charakterisierten Stelle des Mesogastriums befinden, somit weder den Nabel-, noch den Lendenbrüchen angehören.

Die 7 operierten Nabelbrüche waren: 1 Nabelschnurbruch, 3 freie, 1 irreponibler und 2 incarcerierte Nabelbriiche.

Der Nabelschnurbruch betraf cin 1 Tage altes Kind männlichen Geschlechtes. Dasselbe überlebte den operativen Eingriff nicht

Die 6 übrigen Nabelbrüche betrafen durchwegs weibliche Personen.

Die Operation dieser Bruchart bestand bei kleinen Hernien in Bloßlegung und Reposition des Bruchsackes, in Naht der aufgefrischten Bruchpfortenränder, bei großen Brüchen dagegen wurde das Condam in - B runssche Verfahren angewendet: Zwei bis zum Peritonetum reichende, convexe Mautschnitte zu beiden Seiten des Bruches, Exstirpation des Bruchsackes und Naht sämtlicher Schichten, wie bei einer sonstigen Laparotomie.

Der Wundverlauf war, bis auf Fall No. 5, bei welchem sich die IIeilungsdauer wegen einiger Fadeneiterungen verzögerte, ein glatter, per primam intentionem.

Die Operierten hlieben in den 5, zur Kenntnis gelangten Fällen recidivfrei von einer Patientin fehlt jede Nachricht.

Die 6 operierten Ventralbernien betrafen 1 männliches und 5 weibliche Individuen. Die Hernien waren meist an Stellen ron stark gedehnten Narben, nach Laparatomien etc. entstanden. Das Operationsverfabren glich dem bei den epigastrischen IIernien angewendeten. Die Heilung erfolgte bis auf den eine incarcerierte Hernie betreffenden, Fall No. 6 per primam intentionem.

Die Endresultate der Operation sind nur in 3 Fällen bekannt. Dieselben sind radical geheilt.

Von den recht seltenen Lumbalhernien kam an der chirurgischen Klinik nur 1 Fall ror. Diese Bruchart hatto sich ein 5 jähriges Mädchen im 3. Lebensjahre durch Aufstürtzen auf einen Stein, zugezogen. Der Bruch prasentirte sich als mannsfaustgroße reponible Geschwulst in der rechten Londengegend.

Die Radicaloperation gestaltete sich sehr leicht und einfach. Nach 7 Tagen erfolgte Ileilung per primam. Ob jedoch der Erfolg dauernd geblieben, ist nicht bekannt. 
Cher die Endresultate der Radicaloperationen der Lnterleibsbrïche. 127

C. Brüehe des IIypogastriums.

Von den im Bereiche des Hypogastriums auftretenden Brucharten gelangten nur L eisten- und Schenkelbrüche zur Aufnahme. Bei diesen Brucharten, als den allerhäufigsten, wollen wir etwas länger verweilen.

I. Leistenbrüche.

In dem eingangs angefuihrten Zeitraume wurden 221 Leistenhernien operiert. Dieselben verteilen sich auf 201 Personen (179 Männer, 22 Frauen).

Es kamen vor:

\begin{tabular}{rccc} 
rechtsseitige & bei & 119 Personen \\
linksseitige & $"$ & 62 & $"$ \\
beiderseitige & $\#$ & 20 & $"$ \\
\cline { 2 - 4 } Summa & 201 & Personen
\end{tabular}

Die Hernien waren:

$\begin{array}{crrc}\text { erworben } & \text { in } & 206 & \text { Fällen } \\ \text { angeboren } & n & 9 & " \\ \text { Recidive } & n & 6 & n \\ \text { Zusammen } & 221 & \text { Fälle. }\end{array}$

Als Entstehungsursache wurde fast ausnahmslos ein plötzlich, oder continuierlich wirkendes $\mathrm{T} \mathrm{ra} \mathrm{u} \mathrm{ma} \mathrm{angegeben,} \mathrm{Indessen} \mathrm{ist} \mathrm{solchen}$ Anæaben nur in einer sehr geringen Zahl von Fällen eine Bedeutung beizumessen, da doch die meisten Herniologen darin übereinstimmen, dah die Bildung der IFernien durch das Vorhandensein eines offenen Peritonealsackes vorbereitet und durch die Einwirkung eines Traumas ausgelöst wird, weshalb letzteres in der Mehrzahl der Fälle bloß als mittelbares, nichtdie Entstehung, sondern das Hervortreten der Hernie veranlassendes Moment aufzufassen wäre.

Etwa $2 / 3$ sämtlicher Leistenhernien waren freie $(140), 1 / 2$ eingeklemmte (71) und 10 irreponible Brïche.

Als seltenere Arten finden sich verzeichnet: 1. II. interna s. directa (Nr. 134 der freien Hernien, $1 \mathrm{H}$. inguinoproperitonealis (Nr. 52 der incarcerirten Hernien) und 1 II. Littriana (Nr. 56 der incarcericrten Hernien).

Die Dauer des Leidens betreffend wäre anzuführen, daß in den meisten Fällen das Gebrechen bereits eine Reihe von Jahren bestand

Ilinsichtlich der Größe boten die Brüche mannigfache $\Lambda b$ wechslung dar, denn cs kamen walnuB- bis mannskopfgroße Hernion vor. 
Die Weite des Leistencanals verhielt sich durchschnittlich pronortional zur Größe der Vorlagerang und war die äubere Apertur des Leistenkanals in den meisten Fällen für 1-2 Finger passierbar, in einigen noch weiter.

Von Operationsmethoden wurden an der Klinik die jeweilig modernsten geübt; am allerhäufigsten kam jedoch die Ilethode von Bassini und ihre Modification nach Postempski (Rom) in Anwendung, deren Beschreibung wir deshalb bier wiedergeben:

1. Der Hautschnitt verläuft von der Ilöhe der Spina anterior superior und fingerbreit von derselben entfernt, parallel dem Poupartschen Bande, bis zum Beginn des Scrotums. Die zum Vorschein kommendeu subcutanen Venen werden zwischen 2 Sperrpincetten gefaßt und durchschnitten, dann wird das Unterhautbindegewebe durchtrennt und dadureh die $\Lambda$ poneurose des Obliqus externus sowie der äußere Leistenring bloßgelegt.

II. Die $\Lambda$ poneurose des Obliqnus externus wird in der Richtnng des Hautschnittes, vom äußeren Leistenringe bis einige Centimeter über den inneren hinaus, gespalten und der Bruchsack, rom Bruchsackhalse beginnend, teils stumpf mit der Pincette, teils scharf mit dem Messer, von seinen Hüllen (Cremaster und Tunica vaginalis comumnis) und rom Samenstrang, überdies aber auch der Samenstrang von seinen Hüllen (gleichfalls Cremaster und Tunica vaginalis comnis) isoliert, welch letztere Gebilde durchtrennt werden. (Eine vollständige Isolierung nach unten ist nicht unbedingt notwendig, da man nötigenfalls den Bruchsack durchtrennen und den perphieren Teil im Scrotum belassen kann.)

Hierauf wird der Brachsack in einiger Entfernung vom Halse, zwischen 2 Pincetten eröffnet und der Bruchinhalt reponiert. Etwaige Verwachsungen mit dem Inhalt werden gelöst, vorliegende Netzteile, nach Anlegen von Massenligaturen, exstirpiert.

Sodann wird die bloßgelegte Muskelschichte (M. obliquus internus M. transversus und Fascia transversa) in der Richtung und Länge des Aponeurosensehlitzes durchtrennt, der Bruchsackhals gegen den Wundwinkel emporgezogen, an seiner höchsten Stelle mit Dech am pscher Nadel und starkem Faden durchstochen und nach beiden Seiten fest abgeschnürt. Nachdem man die Ligatur geknotet, sieht man noch einmal nach, ob sie kein vorgeschlüpftes Eingeweidestiick einklemmt, und trägt den peripheren Teil des Bruchsackes, $1 \mathrm{~cm}$ vor der Ligatur, mit der Schere ab. Schließlich wird zuerst das eine, danndas andere Ligaturende in eine Nadel gefädelt und unter 
Leitung des Fingersvonder Bauchhöhleaus, etwa $2-3 \mathrm{~cm}$ nach innen vom oberen Wundwinkel, darch die ganze Dicke der Musculatur durchgestochen und über derselben geknotet. Dadurch wird der Peritonealtrichter an eine widerstandsfähige Stelle der Bauchwand befestigt.

III. Während ein Assistent mittelst stumpfen Hakens den Samenstrang (eventuell samt dem aus dem Scrotum emporgehobenen Hoden) nach innen and oben stark abzieht, werden, unter Leitung des linken Zeigefingers, die Ränder der durchtrennten Muskelplatte in ihrer ganzen Dicke (dureh fortlaufende Naht) vereinigt. Man beginnt knapp unterbalb der Stelle, wo der Samenstrang aus der Bauchhöhle hervortritt, da die Erfahrung lehrt, daß derselbe eine Einschnürung an dieser Stelle sebr gut verträgt, und befestigt dann mit derselben Naht den freien Muskelrand an das Poupartsche Band und das Periost des Schambeins. Zum Schlusse vereinigt man sorgfältig (um einer Nekrose vorzubeugen) mittels fortlaufender Nabt die Schnittränder der Aponenrose des Obliquus externus.

IV. Nun wird der Samenstrang a uf dic A poneurose des $O b$ liquus externus gelagert (eventuell der 'Testikel in die normale Lage zurïckgebracht) und die Hautwunde durch fortlaufende (Catgut-)Naht vollständig gesehlossen, so daß der Samenstrang also nur von der Haut bedeckt wird. Tur in manehen Fällen, wo eine Heilung per primam intentionem von vornherein ausgeschlossen erscheint, ebenso auch bei Fettleibigen, wird nach dem Vorschlage Riedels ein kleiner Jodoformgazestreifen in den unteren Wundwinkel eingeführt.

Anber dem Bassini-Postempskischen Verfahren wurde auch recht häufig die Kochersche Methode in ihren verschiedenen Ausführungsweisen geübt.

Die Endresultate der Radicaloperationen konnten - wie es sich von selbst versteht - nicht in sämtlichen Fällen ermittelt werden. Nur bei einem kleinen Bruchteil waren wir in der Lage, eine Nachuntersuchung vorzunehmen, während wir sonst auf die brieflichen Hitteilungen der Operierten angewiesen waren. Es wurden verzeichnet:

Dauererfolge der Radicaloperationen 132 Fälle

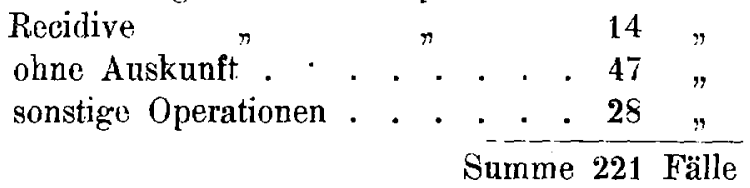

Von Operationsmethoden wurden angewendet: 
Bassini- und Bassini-Postempski 122 mal Kocher II und Kocher III . . . 46 , Sonstige Radicaloperationen . . . . 25 n

Summa 193 Radicaloperationen

In bezug auf diese Operationsmethoden verteilen sich die crzielten Resultate folgendermaBen:

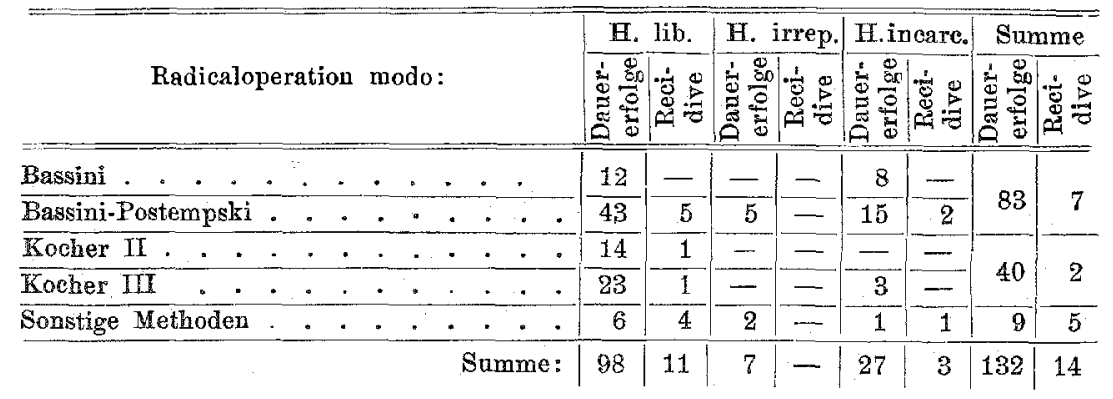

Darnach ergeben sich für die Radicaloperation der Leistenhernien in allgemeinen 9,6 Proz. Yißerfolge. Im speciellen entfallen: auf die Methode von $\mathrm{Bassini}$ und die Modification derselben nach $\mathrm{Po}$ stem pski 7,8 Proz., auf das Kochersche Verfahren 4,9 Proz.Recidive.

Diese Ziffern sprechen sehr zu Gunsten der $\mathrm{K}$ o'ch erschen Verlagerungsmethode, doch müssen wir hervorheben, daß gerade die srößten und ältesten Brïche nach der Bassini-Postempskischen Methode operiert wurden. Auch andere ans verschiedenen Berichten bekannt gewordene Erfolge sind weder für die eine noch für die andere Methode auschlaggebend, sondern liefern summarisch annähernd die gleichen Prozentverhältnisse für beide Methoden.

So veröffentlichten für das Bassinische Verfaluren:

Lebensohn (4) [1898] . . . . . . . . . 2,8 Proz.

Brenner (52) [1898] . . . . . . . . . . . . 5,9 "

Slajmer (9) [1898] (Modifikation nach Wöllfer) . 9,2"

Franz (12) [1899] . . . . . . . . . . . 6,4 "

Coley (28) [1899] . . . . . . . . . . . . . 0,91 "

Andrews (30) [1899] . . . . . . . . . . . 5,0"

Galeazzi (31) [1899]. . . . . . . . . . 5,99"

Bloodgood (27) [1899] (Modifikation vach Halsted) 5,6"

Remedi (32) [1899] . . . . . . . . . . . . 2,42 ,

ILirschk opf (24) [1900] . . . . . . . . . . . . . 4,4,

Hierzu die von Franz (12) im Durchschnitte mit 4,7 "

berechneten Fälle; 
Über die Endresultate bei Radicaloperationen der Cnterleibsbrüche. 131

für das Kochersche Verfahren:

Lebensohin (4) [1898

Bernhardt (29) [1899] . . . . . . . . . . . 15,0 "

Galeazi (31) [1899] . . . . . . . . . . . 5,02

IIirschk of (24) [1900]: laterale Verlagerung . . 2,6 "

(24) [1900]: Invaginationsverlagerung - 1,2 "

Doch betrachten wir beide Methoden noch von anderen Gesichtspunkten aus:

Der postoperative Verlauf gestaltete sich bei beiden Operationsarten annähernd gleich.

Ebenso verhielt es sich mit der Dauer der Heilung. Sie betrug bei beiden Methoden durchschnittlich 7-10 Tage.

Auch in bezug auf Complicationen seitens des Respirationstractes hielten sich beide Methoden das Gleichgewicht.

Graser (23) stellt an eine moderne Operationsmethode folgende Anforderungen :

1. Hohe Unterhindung des. Brucksackhalses, 2. festen Verschlud der Bruchpforte, 3. Heilung per primam intentionem, ohne ausgedehntere Narbenbildung, 4. möglichste Einfachheit des Verfahrens, 5. daß nicht im Dunkel operiert worde.

Welche der beiden Methoden entspricht all diesen Anforderungen vollkommen oder in höherem Maße?

Vollkommen werden diese Postulate von keinem Operationsverfahren erfüllt. Dagngen entspricht die Kochersche Methode den meisten dieser Bedingungen, während ihr in dieser Hinsicht $\mathrm{B}$ assin $\mathrm{i}$ nachsteht. Bei Kocher bleibt nämlich bloß ein Postulat unerfüllt, indem er keinen festen Verschlul3 der Bruchpforte bietet, was indessen nicht von großem Belange zu sein scheint, da doch der Locus minoris resistentiae rregen eine widerstandsfähige Wand verschoben wurde. Bassini hingegen schließt zwar die Brucbpforte fest, gibt aber meist zu ausgedehnter Narbenbildung Anlaß, an welcher Stelle eben die Recidive am häufigsten entstehen. Statt der Lueistenhernie tritt daher oft genug eine Ventralhernie auf, und wir hatten, gelegentlich der Nachuntersuchung, nicht selten Gelegenheit, eine stärkere Vorbauchung der Narbengegend (namentlich beim Husten) wahrzunehmen - das Praeludium zum künftigen Recidiv.

Außerdem entbebrt Bassini der das Kochersche Verfahren besonders auszeichnenden Einfachheit. „Eine sinnreiche, schöne, leichte und sehr einfache Operationsmethode" notiert der begeisterte Operateur in einer der Krankengeschichten.

Während aber die Kochersche Methode sich durch ihre grobe 
Einfacbheit auszeichnet und den Vorteil bietet, daß dabei die Aponeurose des Obliquus externus nicht gespalten wird, besteht ein unschätzbarer Vorteil des Bassinischen Verfahrens darin, daß es bei incarcerierten Hernien die noch immer nach Pierre Franco geübte Terniotomie überflüssig macht.

Grosse (13) betont u. a. als Nachteil der Bassinischen Methode, daß sie zahlreiche Nähte erfordert, daher sehr oft zum Absterben von Gowebsbestandteilen und zu Fadeneiternngen Anlaß gibt, welcher Umstand wieder Methoden ins Leben rief, die das rechtzeitige Entfernen der versenkten Nähte ermöglichen sollten.

Es läbt sich jedoch nicht leugnen, dab das Bassinische Verfabren eine allgemeinere Anwendung als das Kochersche gestattet, welch letzteres Kocher selbst nur "für nicht große und nicht zu dickwandige Brüche“ empfiehlt.

Forschen wir den Ursachen der Recidive nach, so finden wir hauptsächlich folgende: Hohes Alter, Schwäche, große oder complicierte Hernien, Mängel der Anti- oder Aseptik und daraus resultierende Infection, sehlieblich Husten, ja selbst (forciertes) Lachen.

Aus den Ursachen der Recidive lassen sich leicht die Indicationen zur Radicaloperation ableiten.

Im Jahre 1888 stellte Socin (37) für die Radicaloperation der freien Brüche nur eine Indication auf, und zwar die erfolglose Bruchbandbehandlung. Die im abgelaufenen Decennium erzielten günstigen Erfolge jedoch gestatteten es, für die Indicationen immer weitere Grenzen zu ziehen.

Es gelten heutzutage im allgemeinen folgende Indicationen:

1. Erfolglose Bruchbandhehandlung;

2. Irreponibilität oder wiederholte Incarceration;

3. Große Beschwerden, der Wunsch des Patienten und sociale Gründe.

Contraindiciert ist dic Operation bei gestörtem Allgemeinbebefinden und Krankheiten lebenswichtiger Organe.

Wenn auch das hohe Alter keine specielle Gegenanzeige zur Vornahme der Operation bietet, so ist doch letztere mit Rücksicht auf die fast sicher zu erwartenden Recidive in vorgerücktem Alter besser zu unterlassen.

Von Kindern wurden an unserer Klinik sehr wenige operiert, da.nach unserer Erfahrung die meisten Brüche im Kindesalter darch gute und gut beaufsichtigte Bruchbandbehandung zu heilen sind.

Die meisten Autoren (Wirt (38), Coley (39), Iaab (40), Ka- 
Über die Endresultate bei Radicaloperationen der Unterleibsbrüche. 133

rewski (44), Eccles (45), Bitt ner (47) etc.) schildern die Erfolge der an Kindern ausgeführten Radicaloperationen als überaus günstig, empfehlen hierzu die einfachsten, kürzest dauernden Methoden und stellen folgende Indicationen auf:

1. Erfolglose Bruchbandbehandlung;

2. Irreponibilität oder Incarceration;

3. Complication mit Hydrocele, Ectopia vesicae s. ovarii etc.;

4. Sociale Gründe.

Der Zeitpunkt, wan n operiert werden soll, wird verschieden angegeben: er schwankt zwischen dem 2. und 5. Lebensjahr. Fraenkel (48) plaidiert sogar für die radicale Operation im Verlaufe des 1. Lebensjahres, nur müssen eventuell bestehende Darmkatarrhe wesentlich gebessert sein.

Bei incarcerierten Hernien wurden nachstehende Operationen ausgeführt:

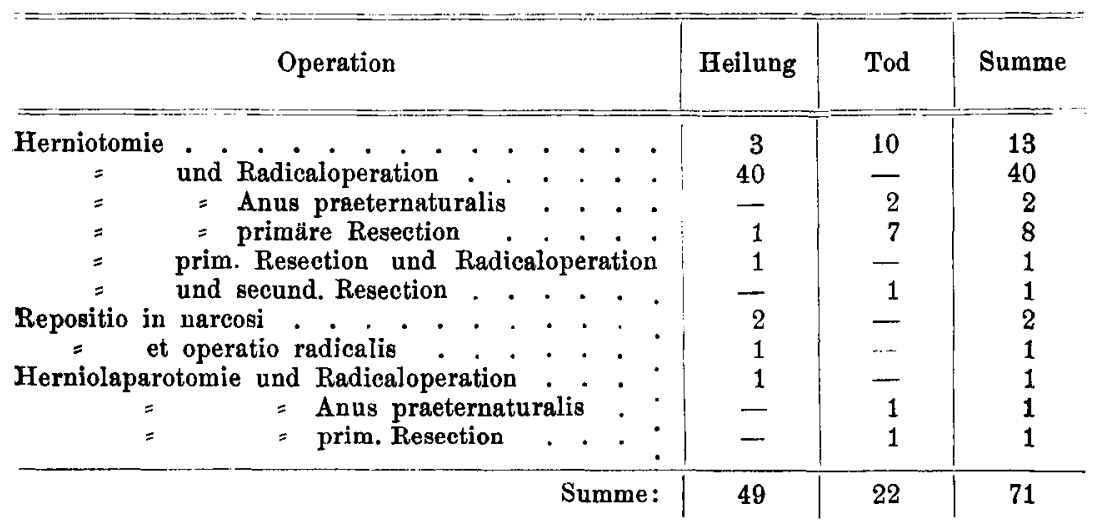

Die hohe Mortalitätsziffer (31 Proz.) findet darin ihre Erklärung, daß die meisten Kranken erst nach mehrtägigem Zuwarten und nach diversen, von unberufenen Personen (Badern, Naturärzten, "Zauberern" und sonstigen Kurpfuschern) resultatlos unternommenen Repositionsversuchen die Klinik aufsuchten, wo sie total erschöpft oder bereits vom Tode angehaucht anlangten.

\section{Schenkelbrüche.}

Die an der chirurgischen Klinik operierten 51 Schenkelbrüche betrafen 49 Personen, und zwar 6 Männer und 43 Frauen.

Die Brüche waren:

27 mal rechtsseitig;

$20 "$ linksseitig;

$2 \%$ beiderseitig. 
Die Erfolge der Radicaloperationen, die in 26 Fällen ausgeführt wurden, müssen als vorzügliche bezeichnet werden, da auf 23 bekannte Fälle blob 1 Recidiv (4,2 Proz.) entfällt.

Leider finden wir die Methode der Radicaloperation nur in einigen Krankengeschichten verzeichnet, müssen es uns daher versagen, dieses Thema näher zu erörtern.

Da die verschiedenen Methoden fast alle gleich gute Dienste leisten, so erscheint es überflüssig, die eine oder die andere derselben erst besonders zu empfehlen, wiewohl auch unter ihnen die Operationen von Bassini und Kocher die meisten Anhänger aufzuweisen haben, während von plastischen Operationen die Methoden von Salzer (Bildung eines Lappens aus der Fascia pectinea), Sehwartz (Muskellappen aus dem Adductor minor) und Davis (Muskellappen aus dem Pectineus) am häufigsten genannt werden. Schließlich könnte auch das jüngst veröffentlichte Verfahren von Ullmann (51) (Unterhautzellgewebslappen) in hierzu geeigneten Fällen erprobt werden.

Die bei den incarcerierten Schenkelhernien ausgeführten Operationen und deren Erfolge sind nachstehender Tabelle zuentnehmen:

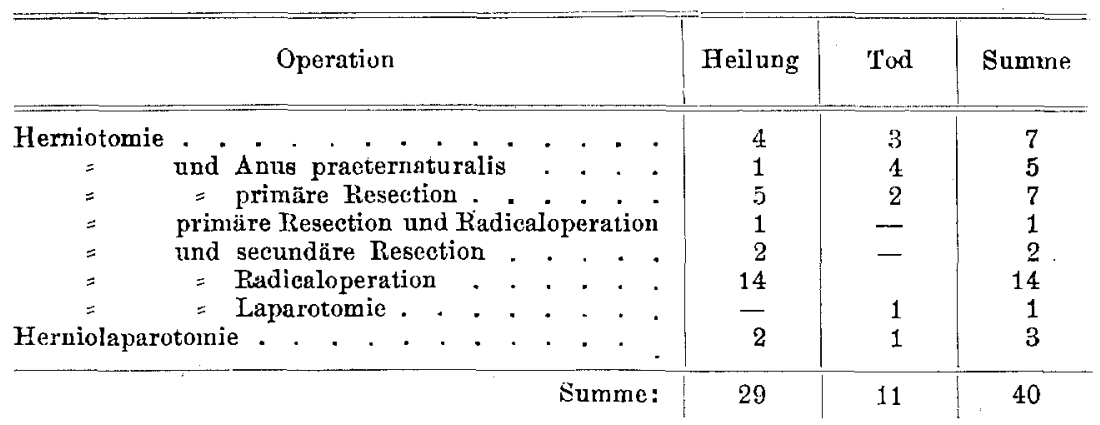

Auch hier gilt für die Mortalitätsziffer (27,5 Proz.) die bei den incarcerierten Leistenbrïchen gemachte Wahrnchmung, daß meist nur hoffnungslose Fälle zur Operation gelangten.

Wir wollen hierbei nicht unterlassen, auf die gefährlichste $\Delta$ rt der eingeklemmten Schenkelbrïche aufmerksam zu machen, und zwar auf die Darmwandbrüche, die unter dem Namen Littrésche Her nien bekannt sind.

Da bei diesen Brüchen nur ein kleiner Teil der Darmwand in der Bruchöffnung liegt, während die gegenüberliegende Wand sich noch in der Bauchböhle befindet, so wird die Einklemmungserscheinung häufig nicht so sehr ausgesprochen und beunruhigend, weshalb diese Incarcerationen in vielen Fällen gar nicht oder $\mathrm{za}$ spät erkannt werden. 
L̈ber die Endresultate bei Radicaloperationen der Lnterleibsbrüche. 135

Zum Schlusse erfüllen wir noch eine angenebme Pflicht, indem wir unserem hochgeehrten Lehrer, Mofrat Prof. R. v. Rydygier und Herrn Prof. Dr. Kader, Direktor der chirurgischen Klinik in Kraka u, für die gütige Erlaubnis, das einschlägige klinische Material zu unserer vorstehenden Arbeit verwerten zu dürfen, an dieser Stelle unseren Dank zum Ausdruck bringen.

\section{Literaturrerzeichnis.}

1) A. Schmidt, Die radicale Hoilung der Unterleibsbrüche nach der Methode des D. C. Sehwalbe. (D. Zeitschr. f. Ch., Bd. 34, S. 90.)

2) Staurenghi, Die Radicalcur der Hernia inguinalis (Allg. Wiener med. Zeitung 1888, p. 237).

3) Frauk, ট̈ъer die Radicaloperation von Leistenhernion. Wien 1893.

4) Lebens ohn, Radicaloperation der Hernien. (D. Ztschr. f. Chir. 1898, Bd. 48 5. und 6. Ireft).

5) Maydl, Die Lehre von den Unterleibsbrüchen, 1898.

6) Berger, Über Unterleibsbrüche, 1897.

7) Winkelmann, Die Lnterleibsbrücho und ihre chiruryische Behandlung, Leipzig 1597.

8) Albert E., Lehrb. der Chir. und Operationslehre 1991.

9) Slajmer E., Bericht über 1 on Radicaloperationen des freien Leistenbruches nach Wölfler (Archiv f. klin. Chir., Bd. 56, Heft 4, 1895.

10) Poullet, Guérison des hernies inguinales et crurales par l'autoplastic tendineuse. (Congrès français de chir. 1896, ref. im Cbl. f. Chir. 1897/ , p. 208.)

11) Poullet, Guérison des hernies inguinales et crurales par l'autoplastic tendineuse. (Congrès français de chir. 1896, ref. im Cb]. f. Chir. 1897, p. 209.)

12) Frank, Herniologische Beobachtungen. (D. Zeitsehr. f. Chir., Bd. 51, 1. und 2. Heft 1899.)

13) Grosse, I., Zur Radicalbehandiung der Leistenhernien. (D. Ztschr. f. Chir. Bd. 57, 1. und 2. Ileft 1900).

14) Brenner. (Cbl. f. Chirurgie 1598, 41.)

15) Borchardt-Körte, ̈̈ber die 'Trendelenburg-Kraskesche Operation. (Beiträge zur klinischen Chirurgie Bd. 20, Heft 2).

16) Witzel. (Cbl. für Chir. 1900, 17).

17) Assaky. (Münch. med. Wochenschr. 1899, 14).

18) Borchardt, M., Osteoplastischer Verschluß großer Bruchpforten. (Beiträge zur klin. Chir., Bd. 20, 2. Ueft 1898.)

19) II ackenbruch, Osteoplastische Radicaloperation für große Schenkelbrüche. (Beiträge zur klin. Chir., Bd. 11, 1894).

20) Beck, C., $A$ new operation for inguinal hemia. (Med. news 1599 September 16, ref. im Cbi. für Chir. 1900, 8.)

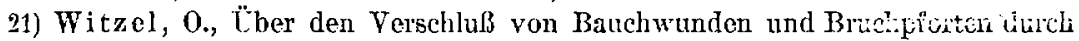
versenkte Silberdrahtnetze (Einheilung von Filigranplatten). (Cbl. für Chir. 1900,10 und 17.) 
22) GoepoI, R., C̈ber die Verschließung von Brachpforten durch Einheilung geflochtener fertiger Silberdrahtnetze (Silberdrahtplatten. (Cbl. für Chir. $1900,17$.

23) Graser, Hernien. (Handbuch dor praktischen Chirurgie von v. Bergmann, v. Bruns und r. Mikulicz, 1900.)

24) Hirsehk opf, Zur Radicaloperation der Hernien. (D. Ztschr. f. Chir., Bd. 56, 1. und 2. Heft 1900.)

25) Nicoladoni, 260 -Radicaloperationen nach Bassini nebst einer eigenen Methode der conservativen Verlagerung des Leistenhodens. (Wiener med. Presse 1895, Nr. 10-17.)

26) Ludwig, Zur Radicaloperation der Leistenbrüche nach Bassini. (Beiträge zur klin. Chir. 1597, Bd. 18, 3. Heft.)

27) Bloodgood, Operations on 459 eases of hernia in the J. Hopkins Hospital from Juni $18 \delta 9$ to January 1899, (Johns Hopkins IIospital Reports 1899: Nr.5-9, ref. im Cbl. für Chin'. 1899, s1).

28) Coley, The radical cure of hernia (Med. news 1599, September 2), ref. im Cbl. für Chir. 1900, 8.

29) Bernhardt, Die Radicaloperation der Leistenbrüche nach Kochers Verlagerangsmethode auf Grund von Erfahrungen der Straßburger chirurgisehen Klinik. (Inaug.-Diss. 1899, ref. im Cbl. f. Chir. 1900, 12.)

30) Andrews, yajor and minor technique of Bassinis operation as performed by himself. (New-York med, record 1599 October 28, ref. im Cbl. für Chir. $1900,12$.

31) Galeazzi, I risultati definitivi nella cura operativa dell' ernia inguinale. (Clinica chirurgica dell' espodale Mauriciano Lmberto I, diretta del Prof. 1. Carle). Estratto dalla clin. chir. 1899 Nr. 6, ref. im Cbl. für Chir. 1901, 4.

32) Remedi, Contributo alla cura radicale delle ernie ed alla patogenesi delle ernie inguinale oblique esterne. (Accademia dei Fisiocritici 1599, ref. im Cbl. für Chir. 1901, 20.)

33) Drobnik, Radicaloperation nael Czorny, Mac Fwen und Bassini (Nowotny lekarskie 1893, 2).

34) Fowler, Annals of Surgery 1897, 59, (ref. in „Medicin der Gegenwart“ 1898, S. 29.)

35) Assaky, (Münch. med. Wochenschr. 1899, S. 437).

30) Rotter, Über die modernen Indicationen zur Radicaloperation freier Hernien. (Borliner klinische Wochenschrift 1899, 7).

37) Socin, Über den Wert der Radicaloperation der Hernien in bezug auf definitive Heilung. (Allg. Wiener med. Zeitg. 1898.)

39) Wirt, Hernien bei Kindern. (Allg. Wiener med. Zeitung 1895, S. 429).

39) Coley. (Archiv of Pediatrics April 1898, ref. in "Medicin der Gegenwart" 1895, S. 433.)

40) Na a B. (Deutsche med. Wochenschr. 1901, 10).

41) Karewski. (Deutsche med. Wochenschr. 1892.)

42) Karewski, Über radicale Bruchoperationon bei Kindern. (Deutsche med. Wochenschr. 1894, 44.)

43) Fracnkel, $\Lambda$., Über Radicaloperation der Leistenbrüche bei Sänglingen. (Cbl. für Chir. 1899, 47.)

44) Ka rewski, Zur Radicaloperation der Leistenbrüche bei Sănglingen. (Cbl. fïr Chir. 1899 , s1.) 
C̈ber die Endresultate bei Radicaloperationen der Unterleibsbrüche. 137

45: Eccles, W. Mc. A dam, The treatment of inguinal hernia in children. (Brit. med. journ. 1899, Y[ai 13, ref. im Cbl. für. Chir. 1899, 51.)

46) Permeau, E. S., Radikaloperationen för icke inklämde bråck utförda å Kronprinsessan Lovisas vårdanstalt för sjuka barn under ằren 1591-159s. (Hygiea Bd. 62, Heft 1, ref. im Cbl. für Chir. 1900, 24).

4i) Bittner W., Über die Radicaloperation des Leistenbruches nebst Bemerkungen zur Ätiologie, Anatomie, Klinik desselben. (Prager med. Wochenschr. 1900,13 , ref. im Cbl. für Chir. 1900, 41).

48) Lenander, K. G., Über die Sensibilität der Bauchhöhle und über die lokale und allgomeine Anästhesie bei Bruch- und Bauchoperationen. '(Cbl. für Chir. 1901, 8.)

49) Bittner, W., Zur :Radicaloperation der Inguinalhernien im Kindesalter. (Archiv für klin. Chir. 1595, Bd. 49, 4. Heft).

50) Tillmanns, Lehrb. der Chir. II. Teil, Bd. 2, 1894.

51) Ullmann, Benützung von Unterhautzellgewebslappen bei : Operationen (Wiener med. Wochenschr. i901, Nr. 46 und 47).

52) Sultan, Atlas und Grundriß der Unterleibsbrüche 1901.

53) Coley, Radical cure of inguinal and femoral herwia, with a report of 845 cases. (Annals of surgery 1901, Juli, ref. im Cbl. für Chir. 1902, 1.)

54) Kocher, Resultate der Hermienradicaloperationen. (Cbl, für Chir. 1897, 19.) 